\title{
Color and visual complexity in abstract images
}

\author{
Rengin Kocaoğlu $\odot$ । Nilgün Olguntürk $\odot$
}

Department of Interior Architecture and Environmental Design, Bilkent University, Ankara, Turkey

Correspondence

Rengin Kocaoğlu, Department of Interior Architecture and Environmental Design, Bilkent University, Ankara, Turkey.

Email: rengin.kocaoglu@bilkent.edu.tr

\begin{abstract}
One of the more important aspects of vision is color perception, which involves aesthetic and psychological responses. The aim of this study is to understand the association between color and visual complexity in abstract images. It is hypothesized that, as the intelligibility of colors in an abstract image decreases, visual complexity and visual interest will increase until there is over unintelligibility where complexity and interest will decrease. In addition, as the difficulty in distinguishing the rules and the variety of colors used in images increases, visual complexity and visual interest will also increase. Abstract images are selected since they are independent from the visual references of the actual world, and are basics of art and architecture. The results revealed that images that were evaluated as visually complex and interesting were the ones that they had difficulty in finding a color harmony and indicate a huge variety of colors to the extent that differentiation between hues becomes very difficult.
\end{abstract}

\section{KEYWORDS}

abstract images, art, color, visual complexity, visual interest

\section{1 | INTRODUCTION}

Everybody experiences color and knows something about it. Most artists, painters, designers and architects work with color intuitively even if they have studied color both practically and theoretically. As Leonardo da Vinci said, "He who loves practice without theory is like the sailor who boards ship without a rudder and compass and never knows where he may cast." thus without understanding color theory and its relationships, a piece would always be missing in the usage of color. Color can conjoin science, mathematics, biology, and technology, like the missing piece, and can be the perfect bridge.

There are many studies from various disciplines on the association between color and emotion, color and aesthetics, color and its naming, and so on. Despite the vast number of separate studies on color and on complexity, there are a limited number of studies on the association between color and visual complexity together. Although the aforementioned developments and studies were quite independent, they have contributed decisively to make the time ideal for an integration that brings the scattered evidence into a global picture. ${ }^{1}$ Having a better understanding of color perception, visual complexity and visual interest can show that different disciplines such as mathematics, science, psychology, design and art have a lot more in common than might be thought.

One of the more important aspects of vision is color perception which involves aesthetic and psychological responses. The model (see Figure 1) created by Leder et al. ${ }^{2}$ introduced the psychological mechanisms involved in the aesthetic appreciation of art which depends on 3 steps; the first is the evaluative dimension, followed by the affective dimension that involves interest and attention, and finally the semantic dimension. The affective dimension, the second step, is important in order to understand the association between visual complexity and colors in abstract images, which induces aesthetic and psychological responses. An understanding of the association between visual complexity and color can help define the relationship between color aesthetics, human vision, and the perception system.

When human vision, and its associated perception system, detects something in its field, that is, a stimulus, it automatically gives a psychological response and makes a judgment about the sense of beauty, which implies the notion of aesthetics. There are several approaches to define aesthetics and one of the most suitable was introduced by Immanuel Kant ${ }^{3}$ in the book "The Critique of Judgment". This approach is a philosophical study of certain states of mind-responses, attitudes and 


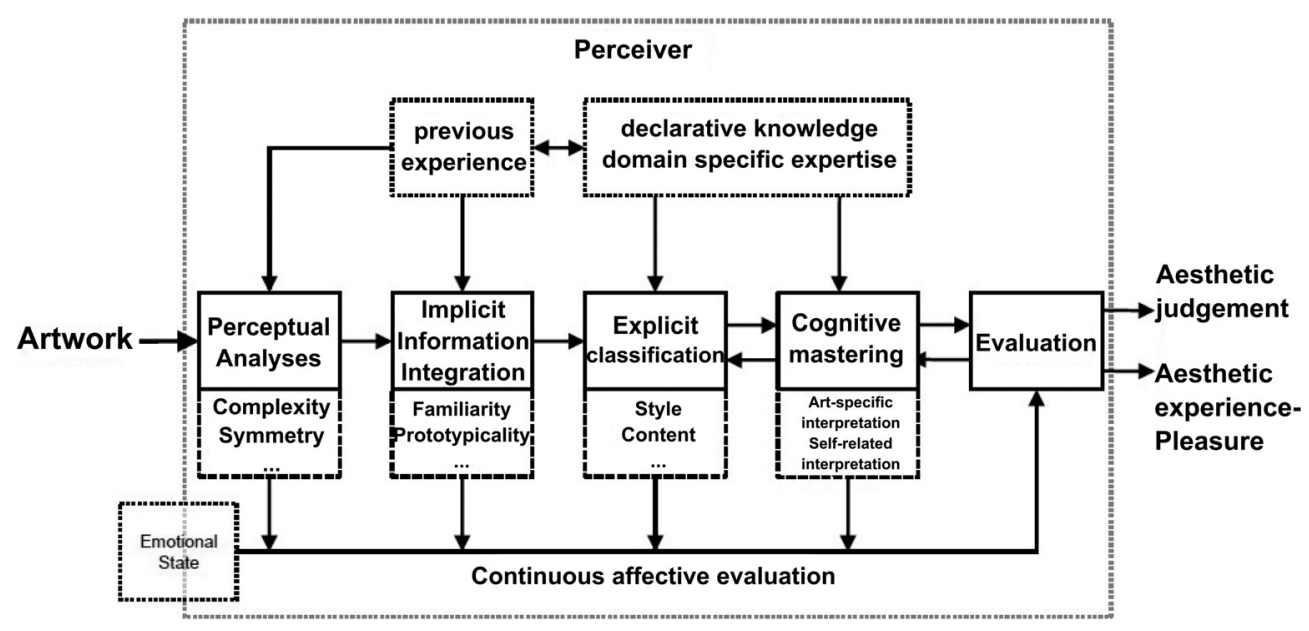

FIGURE 1 Model of aesthetic appreciation and aesthetic judgments (Leder et al., 2004)

emotions - that are held to be involved in the aesthetic experience. Kant located the distinctive features of the aesthetic in the faculty of judgment, whereby humans take up a certain stance toward objects, separating them from their scientific interests and their practical concerns. The key to the aesthetic realm lies therefore in a certain disinterested attitude, which we may assume toward any object and which can be expressed in many contrasting ways. In other words, aesthetic judgment can be defined as the assessment that someone makes of the aesthetic or artistic value of a certain visual stimulus. ${ }^{4}$ Art, as with any other activity of the mind, is subject to psychology, accessible to understanding ${ }^{5}$ and the psychology of art aims to characterize the psychological mechanisms involved in the appreciation of art, such as grasping an artwork's symbolism and identifying its compositional resources. ${ }^{1}$ The aforementioned model (see Figure 1) shows the mental functioning while experiencing aesthetic stimuli. The model aims to provide an integrated description of the psychological processes involved in aesthetic appreciation. It also tries to explain the relation between art and aesthetics, showing the features that make an experience aesthetic, and the relation between contemporary art and more traditional forms of art. ${ }^{1}$ The model is designed to show the relation amongst perception, complexity, familiarity, knowledge, style, content, and experiences (expertise) in the psychological mechanisms involved in the appreciation of art. One of the indicatives for aesthetic judgement is complexity which influences aesthetic evaluation during the early perceptual analysis of objects of aesthetic. ${ }^{6-9}$

Complexity is usually associated with disorder and is defined as the edge of chaos. It is the equilibrator among chaos and ultimate simplicity so it is the transition space between order and disorder. However, this explanation is not enough to understand totally the concept of complexity. There are various definitions of complexity coming from different disciplines including art, design, and architecture and in all, complexity is interpreted as the state of having countless parts that cause difficulty in understanding and interpreting the whole. The concept of complexity is defined by its opposition to simplicity, however it is the amount of detail or intricacy in a composition, for instance, a simple composition (with a small amount of detail) has a minimum level of complexity and a complex composition (with a high amount of detail) has a maximum level of complexity. Thus, with an optimum level of complexity, that is, with enough detail, visual interest can be created. Similarly, in design, complexity is diversity or variety so that visual interest is created. Simple compositions can result in monotony or boredom, whereas complex compositions are prone to produce chaos if a strong order fails to be established. Thus, a successful amount of complexity with a readable order is necessary in design. ${ }^{10}$ Since complexity is the transition space between order and disorder, finding the right balance between simplicity and complexity is important to create compositional interest. It is demonstrated that people prefer a middle level of complexity because simpler visual stimuli tend to be perceived as more aesthetically pleasing, probably due to more fluent mental processing of the stimuli. ${ }^{11}$ But, if it is too simple, people are bored, or if it is too complex, they will be confused. ${ }^{10,12}$ People seek organized complexity, and become disinterested in, or repelled by, low or disorganized complexity. ${ }^{13}$

Previous studies have examined visual complexity from single dimensions such as handling it through symmetry, the number of edges of polygons or color. Some of those studies found the effects of those dimensions on visual complexity and some of them did not. Rump ${ }^{14}$ was trying to find a general factor of preference for complexity even if he could not relate the dimensions (asymmetry, number of elements, and variety of elements) of visual complexity and suggested that a specific dimension should be used in visual complexity studies. He also concluded that people's assessment of the complexity of an image may differ depending on the feature they focus on primarily. ${ }^{14}$ Kreitler, Zigler, and Kreitler ${ }^{15}$ argued that visual complexity was best conceived as a multidimensional concept. In order to begin characterizing the structure of this concept they designed a study which used the 5 complexity dimensions; the heterogeneity of elements, the irregularity of the disposition of the elements, the number of elements, the irregularity of the shape, and the incongruence of the juxtaposition of the elements. ${ }^{4}$ As their results showed, 


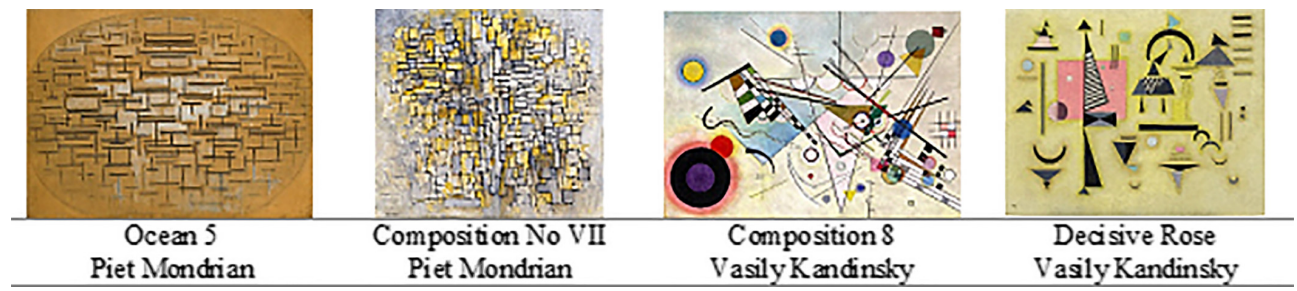

FIGURE 2 The abstract images

a stimulus that facilitates the organization of its meaning and its forms into an overall structure could be rated as simpler than a stimulus with fewer elements, or was more homogeneous, but was harder to integrate or organize into a coherent whole. These findings introduced the view that there could be a multidimensional nature of complexity.

There are 7 dimensions to frame visual complexity; unintelligibility of the elements, disorganization, number of elements, variety of elements, asymmetry, variety of colors, and 3D appearance. ${ }^{4}$ Hence, according to Roberts ${ }^{4}$ the 7 dimensions could have an effect on visual complexity. Amongst all those dimensions, this study focuses only on unintelligibility, disorganization, and variety of colors in a composition since, for examining the association between color and visual complexity, the other dimensions are subdimensions. Unintelligibility is the difficulty in identifying colors in a composition that is, differentiation of colors. If it is not difficult to differentiate colors in a composition, then it is assumed that the visual complexity of that composition is low. The second dimension for visual complexity is disorganization, which is the difficulty in organizing colors in the composition. If there is no discernible rule in the usage of colors; in terms of the location of colors in the composition (symmetrical, radial, diagonal, grid, etc.) and in terms of the harmoniousness of colors (monochromatic, analogous, complementary contrast, split-complementary contrast), the visual complexity of that composition is assumed to be high. The last dimension is the variety of colors (hue, value and chroma) in a composition. As the number of colors increases in a composition, visual complexity also becomes higher.

It is hypothesized that, as the intelligibility of colors in an abstract image decreases, visual complexity and visual interest will increase until there is over unintelligibility where complexity and interest will decrease. When a stimulus is too complex, it is perceived as chaotic, disorganized and unpleasant; if it is too simple, it is perceived as insipid, flat and uninteresting. ${ }^{16}$ In addition, as the difficulty in distinguishing the rules and the variety of colors used in images increases, visual complexity and visual interest will also increase.

\section{2 | METHOD}

\section{1 | Sample group}

The sample group for the study consisted of undergraduate students (second, third, and fourth year) from the Interior
Architecture and Environmental Design Department of Bilkent University, Ankara, Turkey. Each student studied color in their first-year and the total number in the sample group was 120 students. The age range of the students was from 19 to 31 years (mean age 22.5 years) and included both males and females, all with full corrected vision. Also, none of the students indicated they were color defective or color deficient. Each student was randomly assigned to an artwork and only answered one related questionnaire.

\section{2 | Stimuli and apparatus}

To evaluate the association between color and visual complexity, abstract images were selected since they are independent of the visual references to the actual world, and are the basics of art, design and architecture. A visual language is composed in abstract images by just using line, form, shape, and color which makes it universal. Piet Mondrian's 2 abstract paintings; Ocean 5 and Composition No VII, and Vasily Kandinsky's 2 abstract paintings; Composition 8 and Decisive Rose were selected (see Figure 2). These images are equivalent in terms of the quality of lines, shapes, and motives, but are different from each other in terms of visual complexity, especially unintelligibility, disorganization, and variety of colors. The chosen images accomplish color harmony, visual interest, and visual complexity.

Complexity aspects in these abstract images were analyzed with image processing algorithms, and then the colors were identified with the Munsell Color System. This operation could also been done manually since human vision is said to be able to distinguish between 2 and 10 million different colors, but it is not easy to distinguish all colors by the naked eye, even with expert observers. ${ }^{17,18}$ Thus, with image processing algorithms, it is more objective and manageable to identify the colors in the images. This technique is called K-Means Color Clustering. KMeans Clustering is a method of unsupervised learning, which is used when there is unlabeled data (ie, data without defined categories or groups). The goal of this algorithm is to find groups in the data, with the number of groups represented by the variable $\mathrm{K}$ (in this study, the variable $\mathrm{K}$ was 10000 so the algorithm can identify 10000 groups). The algorithm works iteratively to assign each data point to one of $\mathrm{K}$ groups based on the features that are provided. Data points are clustered based on feature similarity. The results of K-Means Color Clustering showed that image Composition 8 had the highest visual complexity in terms of variety of colors. The second highest 
TABLE 1 Results of K-mean and AnOva (4: The most, 1: The least)

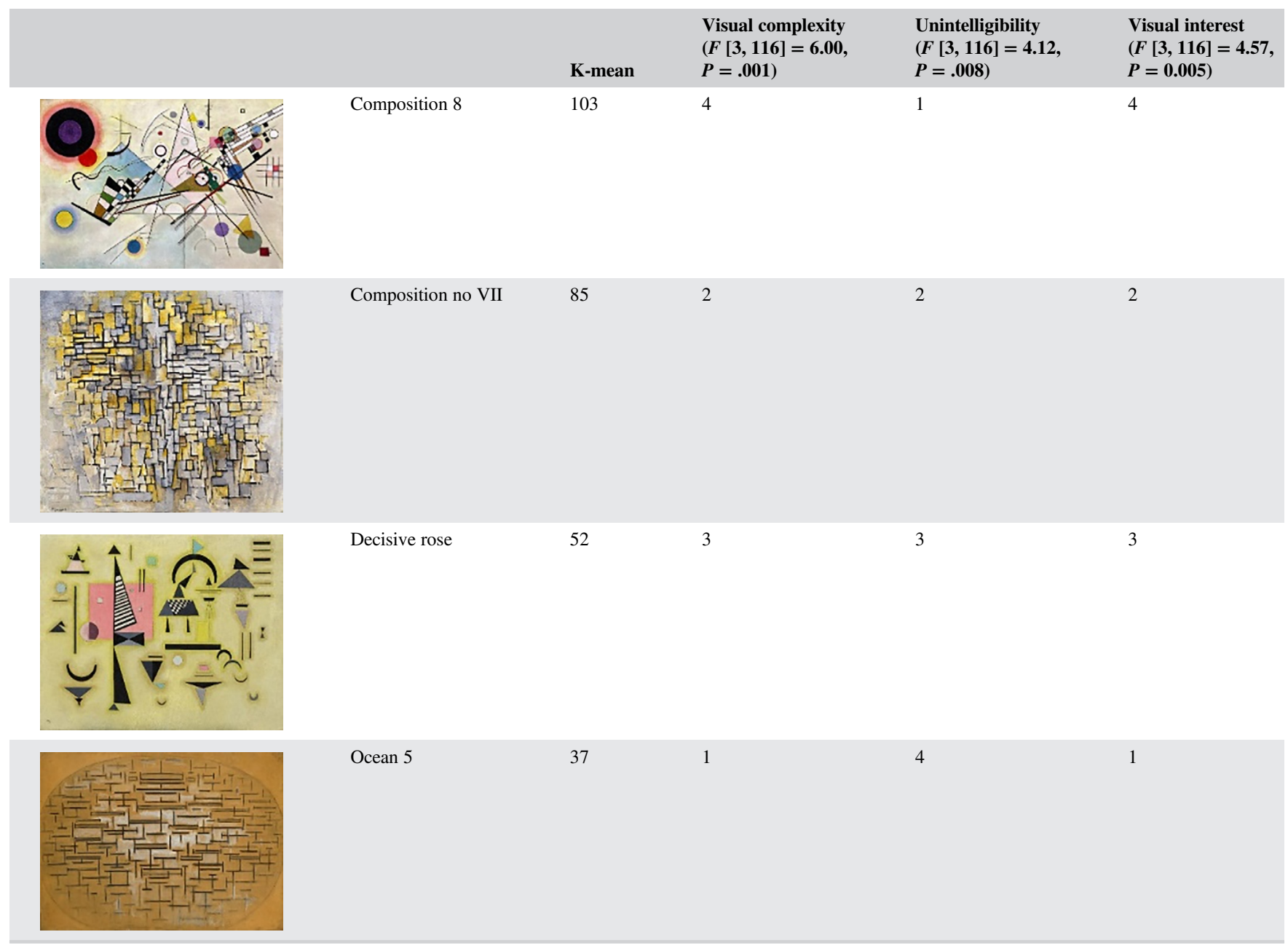

visual complexity was in the image Composition No VII, and the third image was Decisive Rose. The image Ocean 5 had the lowest visual complexity (see Table 1).

After color identification with K-Means Color Clustering in terms of the variety of colors, selected abstract images were rated (with a 5-point scale) by the 120 participants in terms of unintelligibility, disorganization, variety of colors, visual interest and visual complexity. The questionnaire consisted of 5 questions, and the abstract images were printed in color on A4 paper. The first 3 questions were about the dimensions of visual complexity. The fourth question concerned visual interest and the fifth question related to the overall visual complexity in the abstract image. The questions were asked in the subjects' native language in a controlled environment. Since there were 4 different abstract images, each of 30 subjects was randomly assigned to 1 abstract image. Participants were asked to indicate their gender, age and department. Each participant indicated any color vision deficiencies before the experiment and the participants were requested to use their correction vision equipment if required. Each participant voluntarily answered 1 questionnaire where the images were distributed randomly by the researcher. There was no time limitation for answering the questionnaire and participants were free to ask questions.

\section{3 | RESULTS AND DISCUSSION}

The results of the K-Means Color Clustering show that Composition 8 has the highest visual complexity in terms of variety of colors; 103 different colors. The second highest visual complexity was in Composition No VII with 85 colors, and the third was Decisive Rose with 52 colors. Ocean 5 had the lowest visual complexity with only 37 colors (see Table 1). According to the ratings of the participants, Composition 8 had the highest visual complexity, the second highest was Decisive Rose followed by Composition No VII, and the lowest visually complex image was Ocean 5 . Thus, the highest and the lowest visual complex abstract images were the same according to both K-Means Color Clustering and the participants. Also, the association between unintelligibility, disorganization, variety of colors, visual complexity, and visual interest were analyzed according to the results of the participants (see Table 1). According to the results of an analysis of variance (ANOVA) test, no significant difference could be found in terms of disorganization, but there was a significant difference $(F[3,116]=4.12, P=.008)$ between Composition 8, Composition No VII, Ocean 5 and Decisive Rose in terms of unintelligibility. Ocean 5 was rated as the most unintelligible image, followed by Decisive Rose, Composition No VII, 
and Composition 8 respectively. A significant difference could not be found in terms of organizing colors and variety of colors in abstract images. According to the results of the ANOvA test, there was a significant difference $(F[3,116)=4.57, P=$ .005) between Composition 8, Composition No VII, Ocean 5 , and Decisive Rose in terms of visual interest. Composition 8 was rated as the most visually interesting image, followed by Decisive Rose, Composition No VII, and Ocean 5 respectively. Also, there was a significant difference $(F[3,116]=6.00$, $P=.001$ ) between Composition 8, Composition No VII, Ocean 5, and Decisive Rose in terms of visual complexity. Composition 8 was rated as the most visually complex image, followed by Decisive Rose, Composition No VII and Ocean 5 , respectively.

The human visual system has natural limits, for example, the minimal perceivable luminance difference between 2 areas or minimal perceivable dot size. ${ }^{11}$ Such limitations influence the discriminability of information and might cause difficulty in understanding the information in the stimulus even if the amount and organization of the information stays the same. When there is inadequate edge congestion or low figure-ground contrast in the stimulus, there might be uneasiness in identifying the information. The reason why Ocean 5 was rated as the most unintelligible image might be because of these limitations. Ocean 5 has the least number of colors, was rated as the least visually interesting and the least visually complex. On the other hand, Composition 8 was rated as the most intelligible, most visually interesting and most visually complex: also, it had the largest number of colors. This result can be explained by Berlyne's ${ }^{6}$ views for complexity; a stimulus is considered more complex, the larger the number of independently selected elements it contains. In Composition 8, there were more colors than in Ocean 5, also the information in Composition 8 could be identified more easily than in Ocean 5 , since there was a more definite figure-ground relation. Thus, a stimulus can be made more complex by simply multiplying incidents and characters, that is, information. For example, in a painting, the objects depicted, or the areas of uniform color, can be made more numerous and more dissimilar for obtaining a visually complex image. $^{7}$

The results revealed that images that were evaluated as visually complex and interesting were the ones that had less difficulty in differentiating hues (intelligible). Since the selected images are complex in nature, their degrees of complexity revealed that they became over unintelligible that is, when viewers have difficulty in distinguishing hues, they start losing their visual interest.

\section{ACKNOWLEDGMENTS}

This research was conducted as a part of Ph.D. thesis at Bilkent University of the first author, Rengin Kocaoğlu.

\section{CONFLICT OF INTEREST}

The authors declare that they have no conflicts of interest with the contents of this article.

\section{ORCID}

Rengin Kocaoğlu (1D) https://orcid.org/0000-0002-2963-4299 Nilgün Olguntürk (D) https://orcid.org/0000-0001-7947-1101

\section{REFERENCES}

[1] Leder H, Nadal M. Ten years of a model of aesthetic appreciation and aesthetic judgments: the aesthetic episode-developments and challenges in empirical aesthetics. Br J Psychol. 2014;105:443-464.

[2] Leder H, Belke B, Oeberst A, Augustin D. A model of aesthetic appreciation and aesthetic judgments. Br J Psychol. 2004;95:489-508.

[3] Kant I. Critique of Judgment. Indianapolis: Hackett Publishing; 1987.

[4] Roberts MN. Complexity and Aesthetic Preference for Diverse Visual Stimuli [phd thesis]. Spain: Universitat de les Illes Balears; 2007.

[5] Arnheim R. Toward a Psychology of Art. Berkeley, CA: The University of California Press; 1966

[6] Berlyne DE. Novelty, complexity, and hedonic value. Percept Psychophys. 1970;8:279-286

[7] Berlyne DE. Aesthetics and Psychobiology. Appleton-Century-Crofts: New York, NY; 1971.

[8] Gartus A, Leder H. Predicting perceived visual complexity of abstract patterns using computational measures: the influence of mirror symmetry on complexity perception. PLoS One. 2017;12(11):1-29.

[9] Mayer S, Landwehr J. R. When complexity is symmetric: the interplay of two core determinants of visual aesthetics. Adv Consum Res. 2014;42:608-609.

[10] Olguntürk N, Demirkan H. Colour and design: from natural patterns to monochrome compositions. Opt Laser Technol. 2011;43:270-281.

[11] Miniukovich A, De Angeli A. Quantification of interface visual complexity. In Proceedings of the 2014 international working conference on advanced visual interfaces. 2014; 153-160.

[12] Norman DA. Living with Complexity. Cambridge, MA: MIT Press; 2010.

[13] Klinger A, Salingaros N. A. A pattern measure. Environ Planning B: Planning Des. 2000;27:537-547.

[14] Rump EE. Is there a general factor of preference for complexity? Percept Psychophys. 1968;3:346-348.

[15] Kreitler S, Zigler E, Kreitler H. The complexity of complexity. Hum Dev. 1974; 17:54-73

[16] Brighouse G. Variability in preference for simple forms. Psychol Monogr. 1939;51:68-74.

[17] Gouras P. Cortical mechanisms of colour vision. Vision and Visual Dysfunction. London: Macmillan; 1991.

[18] Pointer MR, Attridge GG. The number of discernable colours. Color Res Appl. 1998;23:52-54.

\section{AUTHOR BIOGRAPHIES}

Rengin KoCAOĞLU works as a researcher (PhD Candidate) in Interior Architecture and Environmental Design Department at Bilkent University Ankara, Turkey. Her research interests include perception of color and effects of artificial lighting on attention and performance.

Nilgün Olguntürk is an Associate Professor at the Department of Interior Architecture and Environmental Design, the Faculty of Art, Design and Architecture, Bilkent University, Ankara. She received B.Arch., M.A. and $\mathrm{Ph} . \mathrm{D}$. degrees in architecture. Her professional experience has included appointments as instructor at the Middle East Technical University and research fellow at the London 
South Bank University. She has worked on research projects in the UK for NHS Estates (Department of Health) on color design in hospitals and EPSRC/DTLR LINK (Department of Transport, Local Government and the Regions) on color, visual impairment and transport environments. She has 20 years of research and experience on color perception, color preference and color use in architecture. Her current research and teaching include color and lighting, undergraduate and graduate studios. She is among others, an active member in the CIE (International Commission of Illumination), the AIC (International
Colour Association), the ISCC (Inter-Society Color Council) and the UIA, Chamber of Architects of Turkey.

How to cite this article: Kocaoğlu R, Olguntürk N. Color and visual complexity in abstract images. Color Res Appl. 2018;43:952-957. https://doi.org/10.1002/ $\underline{\text { col. } 22266}$

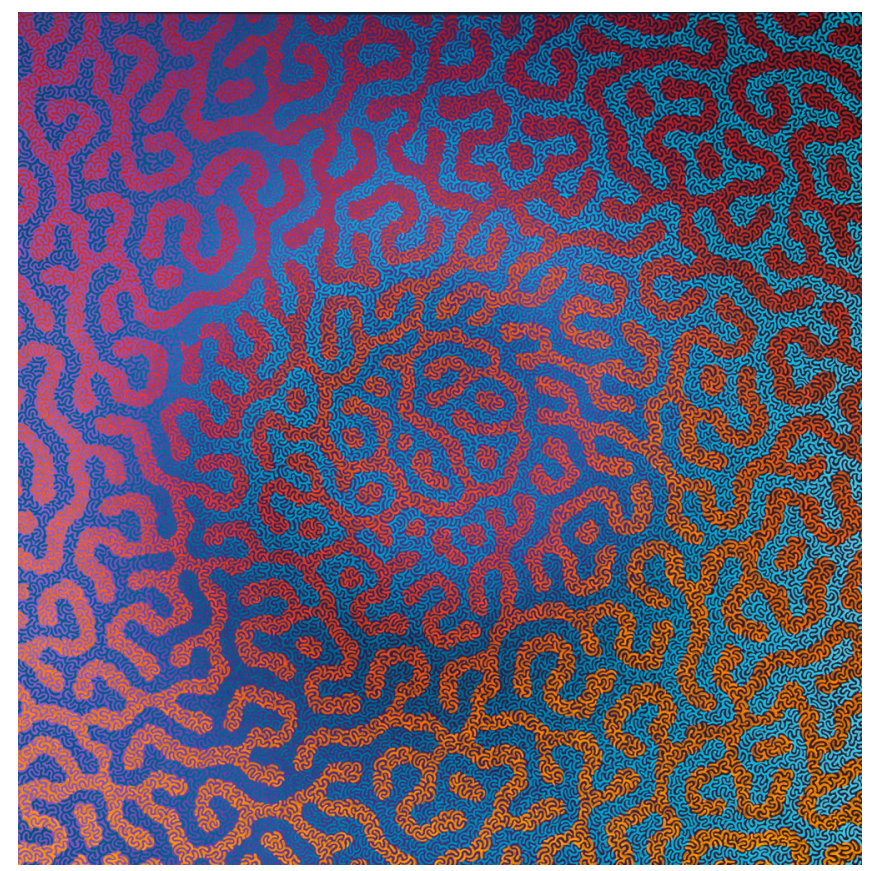

ANDREW WERTH

USA

Title: Elusive \#4

Media: acrylic on canvas 